\title{
A Telerehabilitation Framework for Lower-Limb Functional Recovery
}

\author{
Daniel Cesarini, Pasquale Buonocunto, Mauro Marinoni, and Giorgio Buttazzo \\ Scuola Superiore Sant'Anna, Pisa, Italy \\ \{d.cesarini,p.buonocunto,m.marinoni,g.buttazzo\}@sssup.it
}

\begin{abstract}
Wearable devices are driving the development of post-surgery rehabilitation procedures, also helping in reducing the recovery time and social costs. This paper presents a real-time monitoring framework aimed at supporting telerehabilitation sessions for lower-limbs functional recovery. The presented framework supports patients during the execution of rehabilitation exercises by monitoring the limb movements through a set of low-cost wearable sensors and providing them with multi-modal bio-feedback to enhance the quality of the performed actions. The system also assists the therapist in the definition of exercises tailored to the patient and enables the collection of historical data in cloud-based services for monitoring the effects of therapies and further analysis.
\end{abstract}

\section{Categories and Subject Descriptors}

H.4 [Information Systems Applications]: Miscellaneous

\section{Keywords}

Inertial Measurement Units, Telerehabilitation, bio-feedback, limb tracking, wearable sensors, real-time processing.

\section{INTRODUCTION}

Hospitalization represents a significant monetary cost and source of stress related to post-injury and post-surgery rehabilitation. In fact, the progressive ageing of population is increasing the number of lower limb surgeries, and the demand for rehabilitation will increase over the next decades. The development of mobile and pervasive technologies can give a crucial support for reducing the hospitalization time for patients and the corresponding societal costs, providing new procedures for rehabilitation and telerehabilitation.

The development of telerehabilitation techniques and technologies has at least three positive effects: (i) reducing the workload of the hospitals, thus allowing them to concentrate on the most critical cases; (ii) enabling patients to continue

Permission to make digital or hard copies of all or part of this work for personal or classroom use is granted without fee provided that copies are not made or distributed for profit or commercial advantage and that copies bear this notice and the full citation on the first page. To copy otherwise, to republish, to post on servers or to redistribute to lists, requires prior specific permission and/or a fee.

BodyNets 20142014 London, UK

Copyright 20XX ACM X-XXXXX-XX-X/XX/XX ...\$15.00.

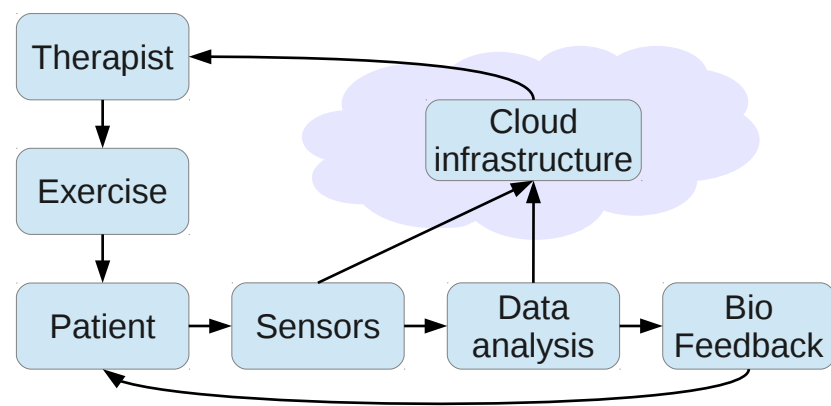

Figure 1: The proposed rehabilitation framework.

their rehabilitation process at home, under specialist supervision; (iii) reducing the overall cost for the society.

Rehabilitation is typically carried out under the supervision of a therapist or physician, who observes the patient and correct the execution of the exercise, if needed. There is a broad range of motor rehabilitation devices [30] that move a limb automatically to follow predetermined trajectories, up to more advanced robotic exoskeletons 25], which leave more freedom to the patient and prevent wrong motions through force and impedance control. These machines are usually required when patients experienced an injury or surgery and need to perform exercises even before they completely recover self-motion. For other cases, or in a second phase of the rehabilitation process, we envision light and affordable systems (based on wearable devices) that support the telerehabilitation sessions under the remote supervision of physicians.

To advance the state of the art in this application field, we present a complete telerehabilitation framework that supports both the patient and the therapist along the rehabilitation path. More precisely, the system supports the therapist in the definition of the exercises specified in terms of postures and trajectories for the specific patient. During rehabilitation, the patient is guided by the system to correctly execute the exercises and, at the same time, is provided with a multi-modal feedback (e.g., visual, haptic, and auditive) to enhance the performance of the exercise. All the data produced during the session are collected and uploaded to a cloud-based infrastructure for storage and further analysis. The result of the analysis is provided to the therapist in order to follow the evolution of the patient and tune the therapy, if needed. An overview of the proposed scenario is illustrated in Figure 1. 
As a reference application for the proposed framework, we address the problem of the knee joint rehabilitation, using a set of wireless motion sensors to track patients actions.

Our framework is based on a distributed systems, composed of: motion sensors, signal processing, data fusion algorithms, feedback generation, communication protocols, cloud-based services, data visualization, and therapy adjustment. Limb motion is reconstructed by integrating the sensory data produced by multiple Inertial Measurement Units (IMUs). Joint angles are then sent via wireless communication to a mobile device, which provides the required feedback to the patient. At the end of the rehabilitation session, the mobile device automatically transfers all data to the cloud service. The therapist or the physician can then retrieve the patients data from the Cloud using a $\mathrm{PC}$ or mobile device, analyse the exercises, and modify the exercises and/or the therapy remotely.

The focus of this paper is the presentation of a complete patient-centric system (from sensors to service). Several research works proposed systems to monitor limbs motion for rehabilitation through IMU sensors 18, 19, while others focused on possibilities of using multi-modal motion based Bio-Feedback to improve skill learning in sports 23. In the field of Interaction Design, some researchers developed a graphical application aimed at easily defining sequences of actions based on sensor signal's interactive processing 15 . Other studies found that the possibility for the users themselves to define when to receive the feedback enhances the quality of motions 8. 9. Inspired by those works and based on our previous experience, we present an application specific framework, that can however be easily extended and reused in different use scenarios.

Paper organization. The rest of the paper is organized as follows: Section 2 describes the framework and its modules, in particular: Section 2.1 presents existing technologies and methodologies used to track movements, analyse them, and provide a feedback and the solution used in the proposed application scenario; Section 2.2 describes the cloud-based infrastructure needed to provide services to both patients and physicians in the proposed telerehabilitation framework; in Section 2.3 are described the services provided to the physician to help him in the definition of the rehabilitation exercises and their supervision. Section 3 presents the open problems in the development of the framework and finally Section 4 concludes the work, providing ideas about future steps and envisioned evolution of the research.

\section{FRAMEWORK DESCRIPTION}

This Section introduces the principal components of the framework. In particular, it is composed of: (i) patient support module; (ii) cloud based services; and (iii) physician support module. Figure 2 shows a high level representation of these components and how they interact.

\subsection{Patient support module}

In this Section we will explore the patient support module, composed of: a motion-sensing system; data processing capabilities; and a task-specific Bio-Feedback system.

\subsubsection{Sensing part}

When monitoring patient movements it is crucial to correctly define the constraints under which to perform the tracking platform selection. These could include issues regarding precision in the measurements, portability, costs, etc.

Technologies and methods that can be used for limb tracking are briefly summarized in Figure 3 In the following, for each technology we briefly describe working principle and respective pros and cons.

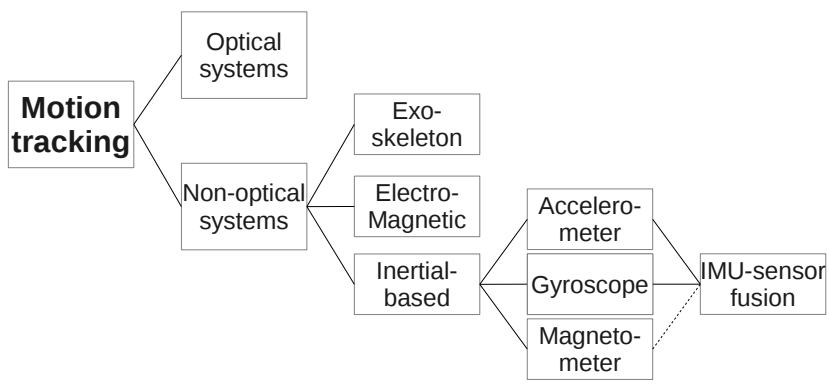

Figure 3: Motion capture system classification, adapted from 10

Optical systems utilize data captured from image sensors to triangulate the $3 \mathrm{D}$ position of a subject between one or more cameras calibrated to provide overlapping projections. Data acquisition is traditionally implemented using special markers attached to a person; however, more recent systems are able to generate accurate data by tracking surface features. These systems produce data with 3 degrees of freedom for each marker, and rotational information must be inferred from the relative orientation of three or more markers. Pros: the markers worn by people are lightweight and robust. Cons: occlusion of markers can occur; the system is bound to the camera infrastructure, not mobile.

Exo-skeleton motion capture systems track body joint angles and are often referred to as exo-skeleton motion capture systems, due to the way the sensors are attached to the body. Mechanical motion capture systems are real-time, relatively low-cost, free-of-occlusion, and wireless (untethered) systems that have unlimited capture volume. Typically, they are rigid structures of jointed, straight rods linked together with potentiometers that articulate at the joints of the body. Pros: accurate, not fixed infrastructure, can provide limited force feedback or Haptic input. Cons: can hinder execution of movements.

Electro-Magnetic systems calculate position and orientation by the relative magnetic flux of three orthogonal coils on both the transmitter and each receiver (placed on users body). The relative intensity of the voltage or current of the three coils allows these systems to calculate both range and orientation by meticulously mapping the tracking volume. The sensor output is 6DOF (Degree-of-Freedom), which provides useful results obtained with two-thirds the number of markers required in optical systems. The markers are not occluded by non-metallic objects but are susceptible to magnetic and electrical interference from metal objects in the environment and electrical sources. Pros: less markers than optical marker-based systems. Cons: metal objects and electrical sources interfere with sensors, the wiring from the sensors can preclude some movements.

Inertial systems: Inertial Motion Capture 24 technology is based on miniature inertial sensors, biomechanical models and sensor fusion algorithms. The motion data of 


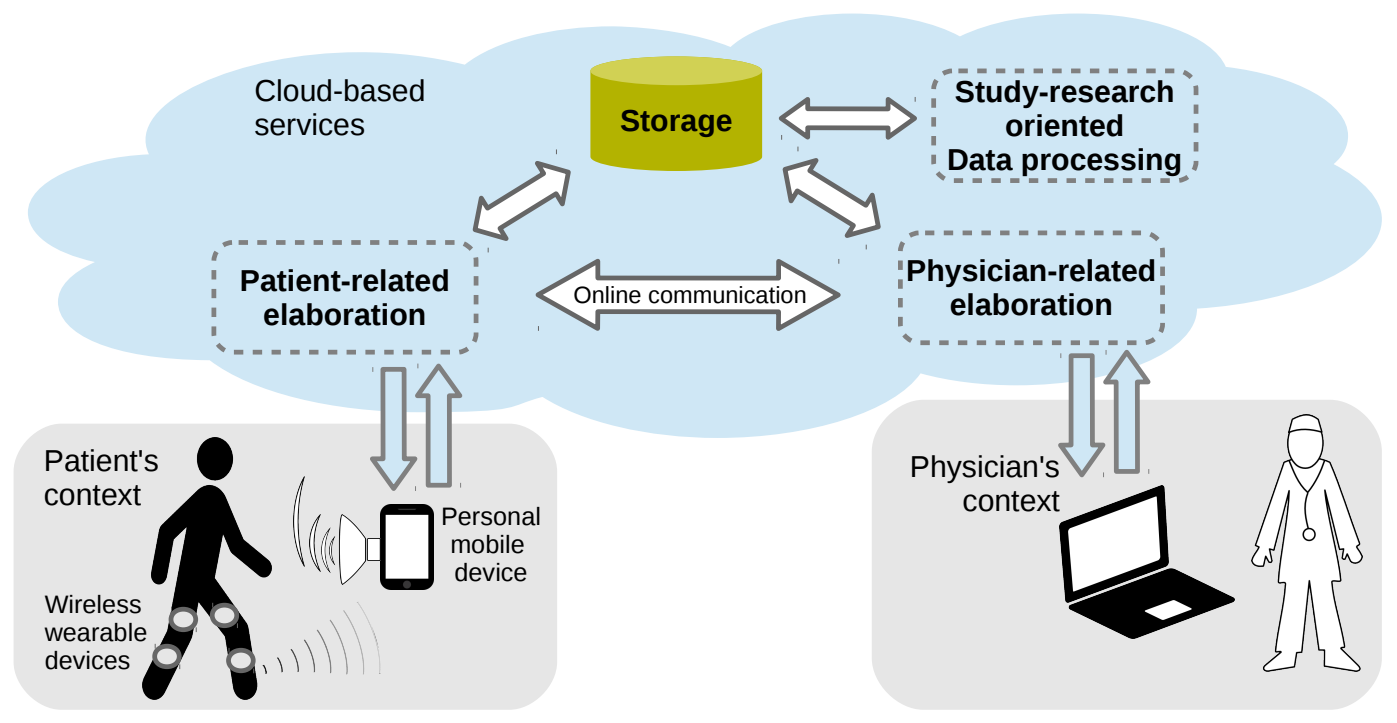

Figure 2: The complete system usage

the inertial sensors can be transmitted (via wireless) to a computer, where the motion is recorded or viewed. Inertial systems use gyroscopes to measure rotational rates, accelerometers to measure accelerations, and magnetometers to measure magnetic natural field. Those signals are merged to obtain more accuracy in estimation of 9DOF, using sensor fusion. Pros: no solving, portability, and large capture areas. Cons: 'floating' where the user looks like a marionette on strings, lower positional accuracy and positional drift which can compound over time.

There are several commercial inertial tracking products available today. Typical full body tracking systems, such as the Moven motion capture suite by Xsens 11 or similar product by Intersense ${ }^{2}$, and many others. They are very expensive, and are not flexible because they cannot be customized and expanded (e.g., adding other kind of sensors), and use proprietary wireless communication protocols that requires special hardware. There are also several academic works involving motion tracking using wireless nodes with on-board IMU. Nevertheless, most of them are highly application specific focusing on data processing rather than on the sensor node hardware and firmware, because they use already available nodes and the output of their sensors.

For these reasons, we decided to design from scratch a low-cost tracking system based on a set of wearable inertial measurement units (IMUs) coordinated as a wireless body area network 4]. Each node monitors a single rigid limb segment and can be extended connecting several additional sensors (e.g., EMG, pressure, temperature).

Nodes send acquired signals to a mobile device (i.e., smartphone, tablet) via a Bluetooth Low Energy (BLE) channel, selected for a number of reasons, among which its widespread adoption, lower power consumption, better immunity to interferences, and net payload data-rate 20].

Body movements are tracked by integrating the angular information acquired from the nodes. A crucial aspect in

\footnotetext{
${ }^{1}$ Xsense MTi - www.xsens. com/products/mti/

${ }^{2}$ Intersense InertiaCube2 - www. intersense.com/pages/18/ $55 /$
}

monitoring limb positions and movements is to guarantee a good level of precision in the measurements. Concerning knee telerehabilitation, the flexion/extension angle estimation is required to have an error lower than 1 degree.

Data are used to present a feedback to the user, so it is crucial to guarantee a bounded delay (i.e., few hundred of milliseconds) between the action performed and the feedback visualization. This is a common requirement shared among a wide range of applications, from gaming to remote teleoperated systems.

Furthermore, the nodes are low-power and characterized by a long lifetime, tiny dimensions and light weight. A long lifetime implies a that the user need only seldom to recharge the nodes. Size and weight are required to be small in order to be comfortable and in order to allow accurate measurements, through a low inertia of the sensor device.

To further increase the system usability, a self-calibration procedure has been devised to help the patient wear the nodes in a more comfortable way without reducing the accuracy of measurements.

\subsubsection{Exercise analysis and evaluation}

This section presents methods that are used to process exercise related signals obtained from the sensing system. In particular we concentrate on the analysis of joint angle signals. In fact, joint tracking, based on signals coming from sensors, is logically separated from performance analysis and data visualization. Our sensing system, presented also in [4], is able to provide us $\alpha_{L}(t)$ and $\alpha_{R}(t)$, respectively the angles of the left and right knee joint at time $t$. Figure 4 presents the basic logic blocks composing the data processing flow.

The joint angles are tracked over time as the patient executes exercises, representing the effects of her actions. Data and signal processing techniques allow to study how the actions are performed, and how much the actual actions deviate from a reference pattern of $\alpha_{R e f}(t)$. Different techniques, appropriate for motion data signal processing and analysis do exist. We coarsely categorize these techniques into frequency domain, time domain oriented, and hybrid. 


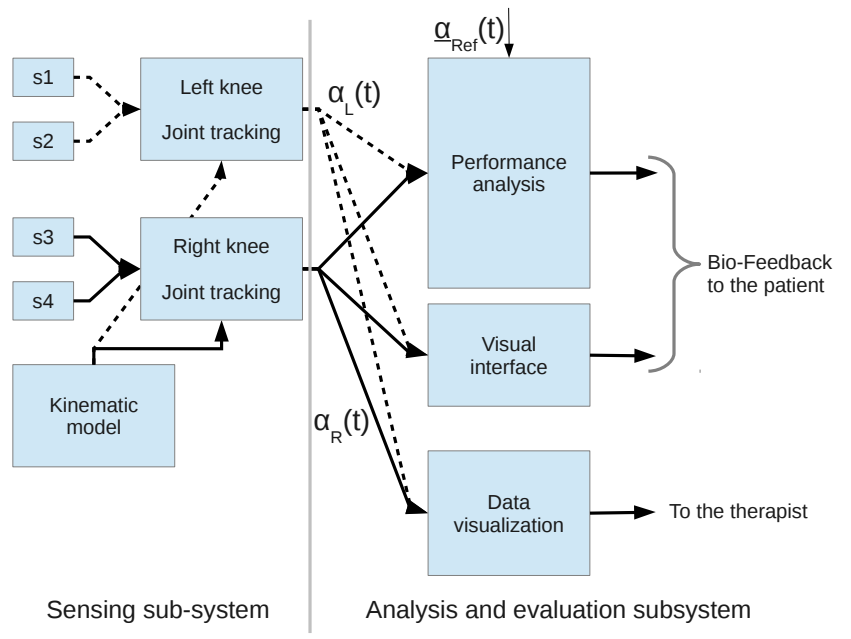

Figure 4: The data processing

Frequency domain. The calculation of the spectrum of a signal allows to get insights into the single frequency components of signals. There are several techniques oriented at calculating the frequency spectrum of signals, based in principle on Fourier transform paradigm (e.g., Fast Furier Transform (FFT), Discrete Fourier Transform, ...), characterized by varying accuracies and computational complexities 31. Frequency analysis can be successfully used to study "hidden" frequency structures of motion (e.g., stroke affected or Parkinsonian patients 28]). However a direct usage of the output of such techniques can be more cumbersome for physicians, for which time-domain analysis offers a more intuitive interpretation.

Time domain. A broad range of time series analysis 13 techniques can be considered as time domain oriented. In order to calculate the degree of similarity of two time series, the most simple calculation is to calculate the euclidean distance between points at corresponding times $t$. Dynamic Time Warping 18, 19 can be considered as an extension to euclidean distance, allowing to compare signals that are shifted and compressed in time with respect to each other. Another appropriate time-domain oriented analysis is statistic; in fact Pearson's cross-correlation has successfully applied to rowing motions, to detect synchronism of different rowers 5 .

Hybrid. Wavelet is a signal processing technique that combines benefits of time and frequency domain 3. In fact wavelet calculates a series of coefficients that are related to both frequency and time 2 . Comparison of these coefficients among the reference signal and the actual signal can provide valuable information. Another class of methods falls within the family of Artificial Intelligence: Forward Neural Networks have been applied to perform diagnoses on Electro-Cardio-Gram (ECG) signals [17. A mixed approach, where wavelet transform, phase-space reconstruction, and Euclidean distance are used to classify normal and epileptic ElectroEncephaloGraphy (EEG) signals is proposed in 21.

What exact motion analysis techniques and methodologies to use in the end system depends upon the exact exercise that needs to be carried out. In fact, if for example an

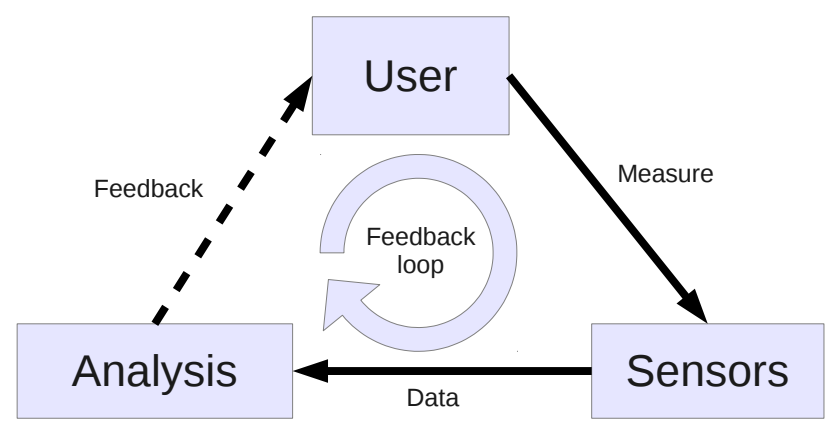

Figure 5: The feedback loop

exercise would be defined as the repetition of 100 times a motion of the joint between $90^{\circ}$ and $120^{\circ}$ a simple threshold based system would be enough. However if we need to perform more complex motion exercises, the usage of DTW or similar techniques would enable a robust comparison of a reference recorded signal (i.e., $\alpha_{\text {Ref }}(t)$ ) with the actual signal $\alpha_{R}$.

\subsubsection{Bio-Feedback}

This Section explains how to provide a Bio-Feedback to the user, based on the outcome of the performed analysis, explained in the preceding section.

Bio-Feedback is thought to provide information back to the user of a system, Figure 5 The development of biofeedback systems demands for at least two concurrent activities: knowledge of existing techniques; and understanding the needs of the specific user groups [5].

Three loops of feedback can be described 11: Exteroceptive feedback, the outcome of the movement through the person's senses, and from external people or devices; Proprioceptive feedback, from proprioceptors in the body and the balance sensors that provide information on the feel of the movement; Kinaesthetic feedback, that is information going to the spinal cord from the limbs and joints, to give information treated at an unconscious level.

An augmented feedback, provided by a human expert or a technical display, effectively enhances motor learning 27, through an enhancement of natural person's feedback loops. A successful feedback, is a feedback that is able to convey information, it is a way of communicating (verbally or non-verbally). In fact, each sense (vision, tact, audition) is able to decode specific environmental stimuli, in a different way. Exploiting the advantages of each sense in the most appropriate way means to become able to design multimodal feedback systems 27]. With respect to the goals of the Bio-Feedback, we can coarsely classify it, in our specific case, into: (a) direct action reporting oriented; (b) anticipatory and motivational oriented; and (c) corrective and error reporting oriented.

The effects of Visual feedback systems if deeply discussed in 14. The effects of visual feedback on altering runners performance is shown in 12. While, regarding Haptic (vibration) feedback systems, a good source of information is 22 .

Regarding Auditory feedback, different communities and research projects provided and provide valuable knowledge: in fact, the design of Auditory displays is a central issue 
in the Sonic Interaction Design (SID) COST Action ${ }^{3}$ while the International Community on Auditory Display (ICAD) is focused on Auditory Displays in general. Auditory Display, in the form of Sonification [16], has been successfully used by one of the authors to present invisible interaction effects, between swimmers hands and water masses, in realtime to both athletes and trainers [6]. A sonification framework geared towards gait problems is presented in 29. That work proposes different mappings [16] for representing actions, and shows how to design a simple to use mapping mixer. It is however missing considerations about how to measure and characterize the "correct" executions and how to represent them acoustically.

As depicted in Figure 5 Bio-Feedback is thought to close the loop of sensing and signal processing, providing valuable information online and in real-time back to the user, in our specific case the patient. As we mainly concentrate on a system that is not mechanically hindering or guiding specific motions, the design of the most adeguate Bio-Feedback methodology is not an easy task 27.

In the following, we analyse the single modes of our multimodal Bio-Feedback ecosystem. The decision about what exact information to map to which feedback quality is postponed to a future experimentation on test subjects. Considering the patients context of the proposed framework as composed of embedded sensing devices (also capable of providing limited audio and vibration stimuli) and a mobile device (capable of displaying 2D, 3D and videos, and of reproducing and synthesizing sounds in real-time) we next analyse how this possibilities are used to compose the multi-modal Bio-Feedback system.

The visual feedback will mainly address the problem of providing information about what to do. More in particular, on the display of the mobile device of the patient we provide a visual feedback, composed of three configurable parts: a $3 \mathrm{D}$ avatar, a video and a $2 \mathrm{D}$ plot. The $3 \mathrm{D}$ avatar can be configured to show either the actual motions performed by the user (using the kinematic information coming from the sensors), or the expected motions, or an integration of the two, through a "ghost" avatar. Along with the avatar we can display a video recorded by the physician when the patient was executing the exercises under his control. A $2 \mathrm{D}$ plot of the actual motion's signal superposed over a goal track, Figure 6. can also represent another way of providing a visual feedback.

The haptic feedback, produced directly on the wearable devices, is used to drive the attention of the user to a particular limb.

The auditory feedback is primarly used (a) to tell how good or bad the patient is performing current task, in real-time and (b) to provide an anticipatory rhythmic pattern to guide the motion. Simplest sonification parameter mappings 16], like a direct pitch mapping, offer the great advantage of a tight coupling between originating phenomenon and the output audible effect. More complex task-oriented mappings represent a bigger hurdle for the user, but provide better results after some training 7 .

The actual composition of the user's visual display in terms of what elements to use (e.g., the avatar, the video) and what information to map to the auditory display sys-

$\sqrt[3]{\text { www.cost.eu/domains_actions/ict/Actions/IC0601 }}$

${ }^{4}$ Official site of the International Community on Auditory Display icad.org/

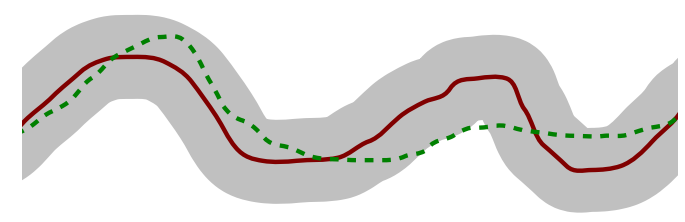

Figure 6: A tracking task. Adapted from 26

tem is left completely configurable, in order to enable the physician to adapt it to the needs of the patient. In fact, we provide to the therapist a tool that allows to compose not only the type of exercises, but also allows to build a personalized feedback scenario, that suits the needs of the user. As an example, consider a children that has to train, it would make sense to build an interface with some visual and auditive stimuli that are attractive for him. On the other hand, a person with some cognitive impairment would probably benefit from a simplified yet informative feedback, to help carrying out the exercises.

\subsection{Cloud services}

The cloud infrastructure is in charge of collecting data from the different actors using the framework and elaborate them in order to provide extra information useful to improve the rehabilitation.

Containing medical data, the communication links between the patients and the server as well as those connecting the physicians need to be protected state of the art security facilities, mandatory for treatment of medical data. Same concerns apply to the storage of acquired data as well as all patients information.

The storage contains different set of information, among them the most important are:

- the archive of all the possible exercises that a physician already prescribed and that can be reused;

- the list of exercises prescribed to a patient;

- the data acquired from the device during each session both with the physician and at home.

To better analyse the stored information, they could be integrated with other patient vital signals and other data from its medical record. In order to have access to those data the system must be able to interoperate with a personal health record (PHR). If some feedback needs to be provided to the patient while he is performing the rehabilitation exercise, also the low-level infrastructure must be able to enforce these timing constraints like proposed in JUNIPER ${ }^{5}$

The collected data can be used to improve the analysis of the patient rehabilitation for evaluation and tuning but can also be elaborated to compare the results from different patients.

Patient-centric elaboration The server could use its computation power to perform a deeper analysis of the data acquired during a session to distil extra information for the physician. It has access also to data from all previous rehabilitation session that can be analysed to compute information to study the patient evolution during the rehabilitation.

${ }^{5}$ JUNIPER Project - http://www.juniper-project.org/ 


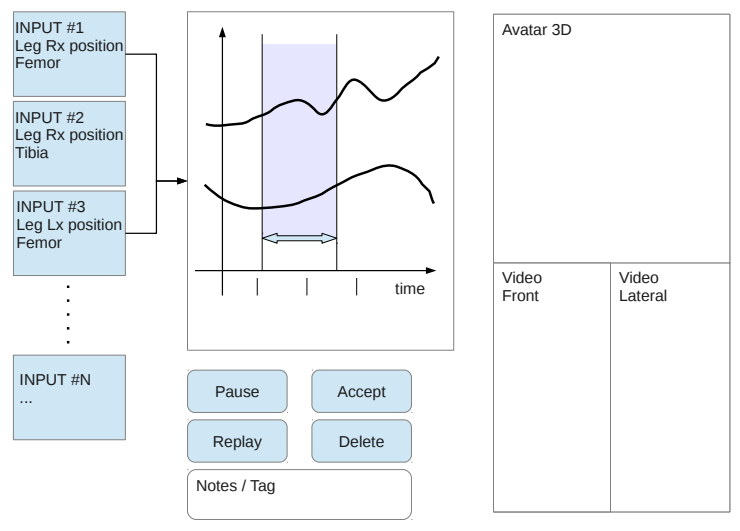

Figure 7: The interface for the caregiver

Inter-patient elaboration Data coming from sessions performed by different patients can be analysed together to provide extra information for the physician. Some examples of the results are the comparison of a patient rehabilitation the detect deviation respect to the average trend, the notification to the physician of exercises which produced good result on patients with similar conditions and the possibility to create a wizard-like approach that simplifies the design of the rehabilitation given the patient initial status and the result of the exercises on similar patients. This kind of analysis can be performed with different machine learning techniques, like the one proposed in 1 .

\subsection{Physician support module}

As we anticipated in the introduction, our framework is supporting the physician on two levels: as an aid during therapy definition; and as an analysis and management tool during the telerehabilitation phase.

Computer Assisted Therapy Definition (CATD). The physician is supported by the CATD software coupled with the sensing systems, worn by the patient, in defining the sets of exercises for the telerehabilitation. In particular, the patient executes the rehabilitation exercises under the supervision of the physician. In the meantime, the physician, using a graphical interface, as the one depicted in Figure 7 selects which kinematic and static magnitudes (e.g., limbs' position, knee angle) are of interest, visualizes them, and selects periods of interest. These periods of interest are tagged accordingly to physicians notes and saved as target motions for the telerehabilitation sessions. As a particular feature of our system, the feedback subsystem can be configured by the physician accordingly to the particular exercises and the specific needs of the patient, e.g., choosing the right stimuli for user categories.

Data visualization / therapy management. During the telerehabilitation period, i.e., when the patient is executing the prescribed exercises, the physician is kept up to date with the achieved results of her patients. The physician visualizes the kinematic values obtained from the sensing system, both as a $2 \mathrm{D}$ plot and as a $3 \mathrm{D}$ avatar, exactly reproducing the patients' limbs motions. Moreover, the physician can remotely adjust the therapy in terms of composition of exercise series (order of execution of movements, number of repetitions, quality of execution goals, etc.). Furthermore, thanks to the inter-patient elaboration module, implemented in the
Cloud, and described in Section 2.2 the physician is supported in studying how different patients evolve in response to the prescribed rehabilitation therapies, in order to improve future rehabilitation and telerehabilitation practices

\section{OPEN PROBLEMS}

The development of the proposed framework opened some issues regarding the topic it addresses. In particular, the most relevant issues regard the analysis of the collected data and the selection of the most appropriate bio-feedback. Regarding the analysis we present two challenges:

- How to evaluate the correctness of the execution of the exercises and how to score the quality of the performance?

- How to aggregate data from different patients and compare their evolution over the time of the therapy?

Furthermore, regarding bio-feedback, we notice that it is hardly arguable that a single one-fits-all feedback does exist, in fact, we also provide following open issues:

- What information to convey to the user?

- Which is the best suited form of feedback for each case?

- When do the effects of the feedback become perceivable by the patient?

- Provided with a bio-feedback, does the patient change the execution of the current cycle, or rather is she changing the motor planning of the next cycle?

\section{CONCLUSIONS}

In this paper we presented a framework to enhance lowerlimb functional recovery through telerehabilitation sessions. The framework comprises a sensing part, based on IMU sensors and data fusion algorithms, a system to provide userspecific Bio-Feedback and a physician related part. In fact, the physician is supported both in the definition of exercises and during the monitoring of the patients evolution. The presented approach can be easily adapted to other fields of application: neurological rehabilitation (e.g., Parkinson, Stroke) and sport training.

\section{REFERENCES}

[1] R. Begg and J. Kamruzzaman. A machine learning approach for automated recognition of movement patterns using basic, kinetic and kinematic gait data. Journal of biomechanics, 38(3):401-408, 2005.

[2] J. Bo, X. Cao, Y. Wan, Y. Yu, P. Hang, P. Mak, and M. Vai. Investigation performance on electrocardiogram signal processing based on an advanced algorithm combining wavelet packet transform (wpt) and hilbert-huang transform (hht)*. In S. Li, Q. Jin, X. Jiang, and J. J. J. H. Park, editors, Frontier and Future Development of Information Technology in Medicine and Education, volume 269 of Lecture Notes in Electrical Engineering, pages 959-968. Springer Netherlands, 2014.

[3] L. Brechet, M.-F. Lucas, C. Doncarli, and D. Farina. Compression of biomedical signals with mother wavelet optimization and best-basis wavelet packet selection. Biomedical Engineering, IEEE Transactions on, 54(12):2186-2192, Dec 2007. 
[4] P. Buonocunto and M. Marinoni. Tracking limbs motion using a wireless network of inertial measurement units. In Proceedings of the 9th IEEE International Symposium on Industrial Embedded Systems (SIES 2014), Pisa, Italy, June 18-20, 2014.

[5] D. Cesarini. Context Aware Computing or the Sense of Context. PhD thesis, University of Pisa, 2014.

[6] D. Cesarini, T. Hermann, and B. Ungerechts. Real-time sonification in swimming: from pressure changes of displaced water to sound. In A. Effenberg, editor, Proceedings of the conference "Multisensory Motor Behavior: Impact of Sound", 2013.

[7] D. Cesarini, T. Hermann, and B. Ungerechts. A real-time auditory biofeedback system for sports swimming. In Proceedings of the International Conference on Auditory Display 2014, Sonification for Sports and Performance Workshop, June 22, 2014 NY, US, 2014

[8] S. Chiviacowsky and G. Wulf. Self-controlled feedback: Does it enhance learning because performers get feedback when they need it? Research Quarterly for Exercise and Sport, 73(4):408-415, 2002.

[9] S. Chiviacowsky and G. Wulf. Self-controlled feedback is effective if it is based on the learner's performance. Research quarterly for exercise and sport, 76(1):42-48, 2005.

[10] M. C. Cuervo, A. Olaya, and R. Salamanca. Biomechanical motion capture methods focused on tele-physiotherapy. In Health Care Exchanges (PAHCE), 2013 Pan American, pages 1-6. IEEE, 2013.

[11] B. Davis, R. Bull, J. Roscoe, D. Roscoe, M. Saiz, R. Curran, and R. Dean. Physical education and the study of sport. Mosby London, 2000.

[12] M. Eriksson, K. A. Halvorsen, and L. Gullstrand. Immediate effect of visual and auditory feedback to control the running mechanics of well-trained athletes. Journal of sports sciences, 29(3):253-262, 2011.

[13] J. D. Hamilton. Time series analysis, volume 2. Princeton university press Princeton, 1994.

[14] R. G. Hamman, I. Mekjavic, A. I. Mallinson, and N. S. Longridge. Training effects during repeated therapy sessions of balance training using visual feedback. Archives of physical medicine and rehabilitation, 73(8):738-744, 1992.

[15] B. Hartmann, L. Abdulla, M. Mittal, and S. R. Klemmer. Authoring sensor-based interactions by demonstration with direct manipulation and pattern recognition. In Proceedings of the SIGCHI conference on Human factors in computing systems, pages 145-154. ACM, 2007.

[16] T. Hermann, A. Hunt, and J. G. Neuhoff. The sonification handbook. Logos Verlag, 2011.

[17] H. G. Hosseini, D. Luo, and K. Reynolds. The comparison of different feed forward neural network architectures for $\{\mathrm{ECG}\}$ signal diagnosis. Medical Engineering $\&$ Physics, 28(4):372 - 378, 2006.

[18] N. Kale, J. Lee, R. Lotfian, and R. Jafari. Impact of sensor misplacement on dynamic time warping based human activity recognition using wearable computers. In Proceedings of the conference on Wireless Health, page 7. ACM, 2012.

[19] J. Kim, S. Yang, and M. Gerla. Stroketrack: wireless inertial motion tracking of human arms for stroke telerehabilitation. In Proceedings of the First ACM Workshop on Mobile Systems, Applications, and Services for Healthcare, page 4. ACM, 2011.

[20] N. P. Konstantin Mikhaylov and J. Tervonen. Performance analysis and comparison of bluetooth low energy with ieee 802.15.4 and simpliciti. Journal of Sensor and Actuator Networks, August 2013.

[21] S.-H. Lee, J. S. Lim, J.-K. Kim, J. Yang, and Y. Lee. Classification of normal and epileptic seizure $\{$ EEG $\}$ signals using wavelet transform, phase-space reconstruction, and euclidean distance. Computer Methods and Programs in Biomedicine, 116(1):10 - 25, 2014.

[22] D. Morris, H. Z. Tan, F. Barbagli, T. Chang, and K. Salisbury. Haptic feedback enhances force skill learning. In $W H C$, volume 7, pages 21-26, 2007.

[23] G. Rauter, R. Sigrist, C. Koch, F. Crivelli, M. van Raai, R. Riener, and P. Wolf. Transfer of complex skill learning from virtual to real rowing. PloS one, 8(12):e82145, 2013.

[24] D. Roetenberg, H. Luinge, and P. Slycke. Xsens mvn: full 6dof human motion tracking using miniature inertial sensors. Xsens Motion Technologies BV, Tech. Rep, 2009.

[25] H. Schmidt, C. Werner, R. Bernhardt, S. Hesse, and J. Krüger. Gait rehabilitation machines based on programmable footplates. Journal of neuroengineering and rehabilitation, 4(1):2, 2007.

[26] R. Schmidt and T. Lee. Motor Learning and Performance, 5E With Web Study Guide: From Principles to Application. Human Kinetics, 2013.

[27] R. Sigrist, G. Rauter, R. Riener, and P. Wolf. Augmented visual, auditory, haptic, and multimodal feedback in motor learning: A review. Psychonomic bulletin $\mathcal{E}$ review, 20(1):21-53, 2013.

[28] Y. Su, C. R. Allen, D. Geng, D. Burn, U. Brechany, G. D. Bell, and R. Rowland. 3-d motion system (" data-gloves"): Application for parkinson's disease. Instrumentation and Measurement, IEEE Transactions on, 52(3):662-674, 2003.

[29] A. V. Torres, V. Kluckner, and K. Franinovic. Development of a sonification method to enhance gait rehabilitation. Proceedings of ISon 2013, 4th Interactive Sonification Workshop, 2013.

[30] R. L. Worland, J. Arredondo, F. Angles, F. Lopez-Jimenez, and D. E. Jessup. Home continuous passive motion machine versus professional physical therapy following total knee replacement. The journal of arthroplasty, 13(7):784-787, 1998.

[31] N. Zhang, Z. Nie, Y. Luo, L. Du, X. Wang, and L. Wang. A reconfigurable overlapping fft/ifft filter for ecg signal de-noising. In Bioelectronics and Bioinformatics (ISBB), 2014 IEEE International Symposium on, pages 1-4, April 2014. 